\title{
Regime de metas de inflação no Brasil: metodologia VAR e análise de funções impulso-resposta
}

\author{
André Luiz Correa \\ Depto. de Economia, FCLAR, UNESP \\ 14800-901, Araraquara, SP \\ E-mail: andrelc@fclar.unesp.br \\ Érika Capelato, \\ Depto. de Economia, FCLAR, UNESP \\ 14800-901, Araraquara, SP \\ E-mail: erika@fclar.unesp.br
}

\begin{abstract}
Resumo: O regime monetário de metas de inflação adotado pelo Brasil em meados de 1999 após uma crise cambial que forçou o abandono da chamada âncora cambial, tem o objetivo, resumidamente, de fazer um anúncio prévio de uma meta numérica para a inflação buscando com isso coordenar a formação de expectativas inflacionárias dos agentes e a fixação de salários e preços. Muitos trabalhos empíricos relacionados à avaliação do regime de metas de inflação no Brasil ou adotando modelos empíricos e simulações matemáticas para estudar a compatibilidade entre o regime de metas de inflação e políticas econômicas de diversos matizes. O presente trabalho apresenta um modelo com o objetivo de analisar a inter-relação dinâmica entre variáveis macroeconômicas no contexto do regime de metas de inflação fazendo uma estimação empírica de um modelo VAR para o período 1999-2013.
\end{abstract}

Palavras-Chave: Vetores autorregressivos (VAR), funções de impulso-resposta, metas de inflação

\section{Introdução}

O regime de metas de inflação pode ser caracterizado como um regime de condução da política monetária baseado no estabelecimento de uma meta para alguma medida de inflação oficial, que será amplamente divulgada pelo governo e cujo cumprimento deve ser responsabilidade do Banco Central $^{1}$. O instrumento de política econômica preferencialmente utilizado para o cumprimento da meta é a taxa de juros. Acredita-se que os mecanismos de transmissão dos juros às demais variáveis são capazes de conduzir a inflação à meta preestabelecida. Em condições ideais de funcionamento, este regime proporcionaria uma maior transparência na condução da política monetária, baseada na busca pelo aprimoramento dos canais de comunicação entre o Banco Central e os agentes. Por conseguinte, as metas podem coordenar a formação de expectativas inflacionárias dos agentes e a

\footnotetext{
${ }^{1}$ Em [10] podem ser encontradas discussões sobre distintos regimes monetários e referências adicionais sobre o tema.
} 
fixação de salários e preços, operando como uma âncora nominal tanto para a inflação atual como para as expectativas futuras.

Diversos autores das mais variadas orientações teóricas em economia tem discutido os resultados do regime de metas de inflação após sua implementação e operacionalização em meados de 1999. As primeiras discussões focaram em aspectos técnicos do modelo, com destaque para os trabalhos de [3] e [9]. Após o período de implementação, e verificada a relativa consolidação do regime, houve um aumento substancial no número de trabalhos empíricos relacionados à avaliação do mesmo. Grande parte dos textos adota uma posição crítica ao regime de metas, como por exemplo [11]. Outra vertente de trabalhos tem procurado avaliar, por meio de modelos e simulações matemáticas, a compatibilidade entre o regime de metas de inflação e políticas econômicas de natureza heterodoxa, como por exemplo [7], [8] e [12]. Todavia, os modelos presentes nestes trabalhos não exploram explicitamente variáveis associadas à questão fiscal ou ao setor externo, como a taxa de câmbio. Adicionalmente, não são efetuados testes empíricos.

No caso brasileiro, as autoridades monetárias brasileiras escolheram um índice de preços cheio como meta para inflação, neste caso o IPCA (Índice de Preços ao Consumidor Amplo, calculado mensalmente pelo IBGE e que compreende famílias com renda até 40 salários mínimos). Em alguns países que adotam o regime de metas de inflação, a escolha recaiu sobre alguma medida de núcleo da inflação, que tem como propriedade excluir ou diminuir o peso de choques de preços provocados por fatores temporários ou casuais, reduzindo eventuais picos de volatilidade. Por conseguinte, um aumento generalizado de preços na economia estaria refletido no núcleo, porém, um aumento localizado no preço de algum item específico, por conta de qualquer evento esporádico, seria excluído. Não obstante, a definição do núcleo da inflação pode não ser facilmente compreensível para os agentes, enfraquecendo a credibilidade do regime.

Neste trabalho propomos a estimação empírica de um modelo de séries temporais relacionando a medida de inflação oficial com uma medida do diferencial de juros doméstico em relação ao juros externos, a taxa de câmbio, que historicamente se apresenta como elemento controverso no debate econômico e uma medida de hiato do produto. De acordo com as funções de impulso-respostas, calculadas a partir da metodologia VAR, pretende-se analisar as respostas das variáveis escolhidas a choques nas demais variáveis consideradas no modelo.

\section{Metodologia}

A metodologia VAR (Vetores Autorregressivos), desenvolvida a partir do trabalho [13], representou um avanço no processo de estimação de modelos econômicos 
multivariados, até o momento realizada principalmente por meio de modelos de equações simultâneas. De maneira geral, podemos expressar um modelo autorregressivo de ordem $p$ com $n$ variáveis endógenas $\mathbf{X}_{\mathbf{t}}$ de acordo com (1):

$$
\mathbf{A} \mathbf{X}_{\mathbf{t}}=\mathbf{B}_{\mathbf{0}}+\sum_{i=1}^{p} \mathbf{B}_{\mathbf{i}} \mathbf{X}_{\mathbf{t}-\mathbf{i}}+\mathbf{B} \boldsymbol{\varepsilon}_{\mathbf{t}}
$$

Onde A é uma matriz $n \times n$ que define as restrições contemporâneas entre $\mathrm{s}$ variáveis; Xt é um vetor de variáveis $n \times 1 ; \mathbf{B}_{0}$ é um vetor de constantes; $\mathbf{B}_{\mathbf{i}}$ são matrizes $n \times \mathrm{X}$ $n$, $\mathbf{B}$ é uma matriz diagonal de dimensão $n$ e $\varepsilon$ é um vetor de choques aleatórios, de modo que:

$$
\boldsymbol{\varepsilon}_{t} \sim \text { i.i.d }\left(\mathbf{0} ; \mathbf{I}_{\mathbf{n}}\right)
$$

A condição de estabilidade do sistema é que todos os valores de $L$ que satisfaçam (3) estejam fora do círculo unitário.

$$
\left|\mathbf{I}-\mathbf{B}_{1} L-\ldots-\mathbf{B}_{p} L^{p}\right|=0 .
$$

Comumente o modelo (1) é estimado em sua forma reduzida

$$
\mathbf{X}_{\mathbf{t}}=\mathbf{A}^{-\mathbf{1}} \mathbf{B}_{\mathbf{0}}+\sum_{i=1}^{p} \mathbf{A}^{-1} \mathbf{B}_{\mathbf{i}} \mathbf{X}_{\mathbf{t}-\mathbf{i}}+\mathbf{A}^{-1} \mathbf{B} \boldsymbol{\varepsilon}_{\mathbf{t}}=\Pi_{0} \mathbf{B}_{\mathbf{0}}+\sum_{i=1}^{p} \Pi_{\mathbf{i}} \mathbf{X}_{\mathbf{t}-\mathbf{i}}+\Pi \mathbf{B} \boldsymbol{\varepsilon}_{\mathbf{t}}
$$

Onde $\Pi_{0}=\mathbf{A}^{-1} \mathbf{B}_{0} ; \Pi_{i}=\mathbf{A}^{-1} \mathbf{B}_{i} ; \Pi=\mathbf{A}^{-1} \mathbf{B}$.

Todavia, a identificação do modelo torna necessário impor algumas restrições aos coeficientes da matriz A. [13] sugere que as restrições impostas tenham alguma relação com argumentos econômicos. Uma maneira de impor restrições diz respeito à decomposição de Cholesky. A decomposição de Cholesky impõe restrições triangulares, de modo que determinadas variáveis tenha efeito sobre outras em instantes diferentes de tempo. Por exemplo, uma variável é escolhida para ter efeito contemporâneo sobre as outras, a seguinte tem efeito em $t$-1e assim sucessivamente.

Não obstante, em geral os pesquisadores estão menos interessados em estimar os parâmetros do sistema e bastante interessados nas interrelações entre variáveis incluídas no modelo. Para tanto, utiliza-se como ferramenta a Função Impulso-Resposta. Caso o sistema atenda as condições de estacionariedade, $\mathbf{X}_{t}$ possui uma representação em função dos valores passados de $\boldsymbol{\varepsilon}_{t}$ : 


$$
\mathbf{X}_{\mathbf{t}}=\mathbf{C}_{\mathbf{0}}+\boldsymbol{\varepsilon}_{\mathbf{t}}+\sum_{i=1}^{p} \boldsymbol{\Psi}_{\mathbf{i}} \boldsymbol{\varepsilon}_{\mathbf{t}-\mathbf{i}}
$$

Onde $\mathbf{C}_{\mathbf{0}}$ contém as médias do processo e $\boldsymbol{\Psi}(L)=[\boldsymbol{\Phi}(L)]^{-1}$, em que $L$ é o operador defasagem.

Os elementos da matriz $\Psi_{\mathbf{i}}$ correspondem a multiplicadores de impacto de uma mudança em $\boldsymbol{\varepsilon}$ sobre as variáveis endógenas. ${ }^{2}$

\section{II.2 Caracterização dos dados}

Para fins de estimação, consideremos

$$
X_{t}^{\prime}=\left[\begin{array}{llll}
y_{t} & i_{t} & e_{t} & \pi_{t}
\end{array}\right]
$$

Onde $\pi_{t}$ e taxa de inflação correspondente a variação do IPCA em \% no período $t ; e_{t}$ é a variação da taxa cambial para venda em R \$/US\$, disponibilizada pelo Banco Central do Brasil no período $t$ em relação ao período $t-1 ; i_{t}$ é uma medida do diferencial da taxa de juros entre o Brasil e o exterior, definido como: $i_{t}=r^{d}-r^{f}$, em que $r^{d}$ é a taxa Selic (divulgada pelo Banco Central do Brasil) e $r^{f}$ é a taxa de juros dos fundos federais (FED Funds), disponibilizada pelo Federal Reserve no período $t$ e $y_{t}$ é o hiato do produto obtido a partir da aplicação do filtro HodrickPrescott $^{3}$ aos dados do Produto Interno Bruto (PIB) calculados pelo Banco Central do Brasil no período $t$. Uma interpretação simples para $y_{t}$ neste trabalho, consiste em entendê-lo como o desvio do PIB de sua tendência de longo prazo. Os dados utilizados compreendem dados mensais para o período de julho de 1999 a outubro de 2013, totalizando 172 observações.

Antes de iniciar a estimação do modelo VAR conforme os procedimentos sugeridos em [2], verificou-se a estacionariedade ${ }^{4}$ das séries selecionadas através dos testes de estacionariedade augmented Dickey-Fuller - ADF e KPSS. A estatística de teste apresentada na tabela baseia-se no modelo selecionado através dos critérios de informação (Akaike e Bayesiano) sendo escolhida por meio de estimações recursivas a partir de 13 defasagens. ${ }^{5}$

\footnotetext{
${ }^{2}$ Para maiores detalhes ver [6].

${ }^{3}$ Para maiores detalhes sobre Filtro de Hodrick-Prescott ver [2].

${ }^{4}$ As consequências da utilização de séries não estacionárias pode ser encontrada em detalhes em [2] e [6].

${ }^{5}$ Os resultados completos não são apresentados por conta das limitações de espaço.
} 
Tabela 1 - Testes de estacionariedade para as variáveis selecionadas.

\begin{tabular}{lll}
\hline Variável & ADF & KPSS \\
\hline$\pi$ & estacionária & estacionária \\
$e$ & estacionária & estacionária \\
$y$ & estacionária & estacionária \\
$i$ & não estacionária & não estacionária
\end{tabular}

Fonte: elaboração própria.

Os resultados da Tabela 1 sugerem que as variáveis $\pi$, $e$ e $y$ devem ser utilizadas em nível, enquanto a variável $i$ deve ser tomadas em primeiras diferenças.

A seguir, determinou-se o número de defasagens do modelo VAR, iniciando a análise em 13 defasagens. Para o modelo (1) os resultados, de acordo com o Critério de Informação de Schwarz, indicaram uma defasagem.

Os gráficos das funções de impulso-resposta são apresentados abaixo. Conforme destaca [2], a aplicação de um choque (no presente caso da magnitude de um desvio padrão) a uma das variáveis afeta todas as variáveis do sistema em decorrência das interações dinâmicas. A ordenação de variáveis adotada foi:

$$
y \rightarrow \pi \rightarrow i \rightarrow e .
$$

Figura 1 - Funções impulso-resposta. 

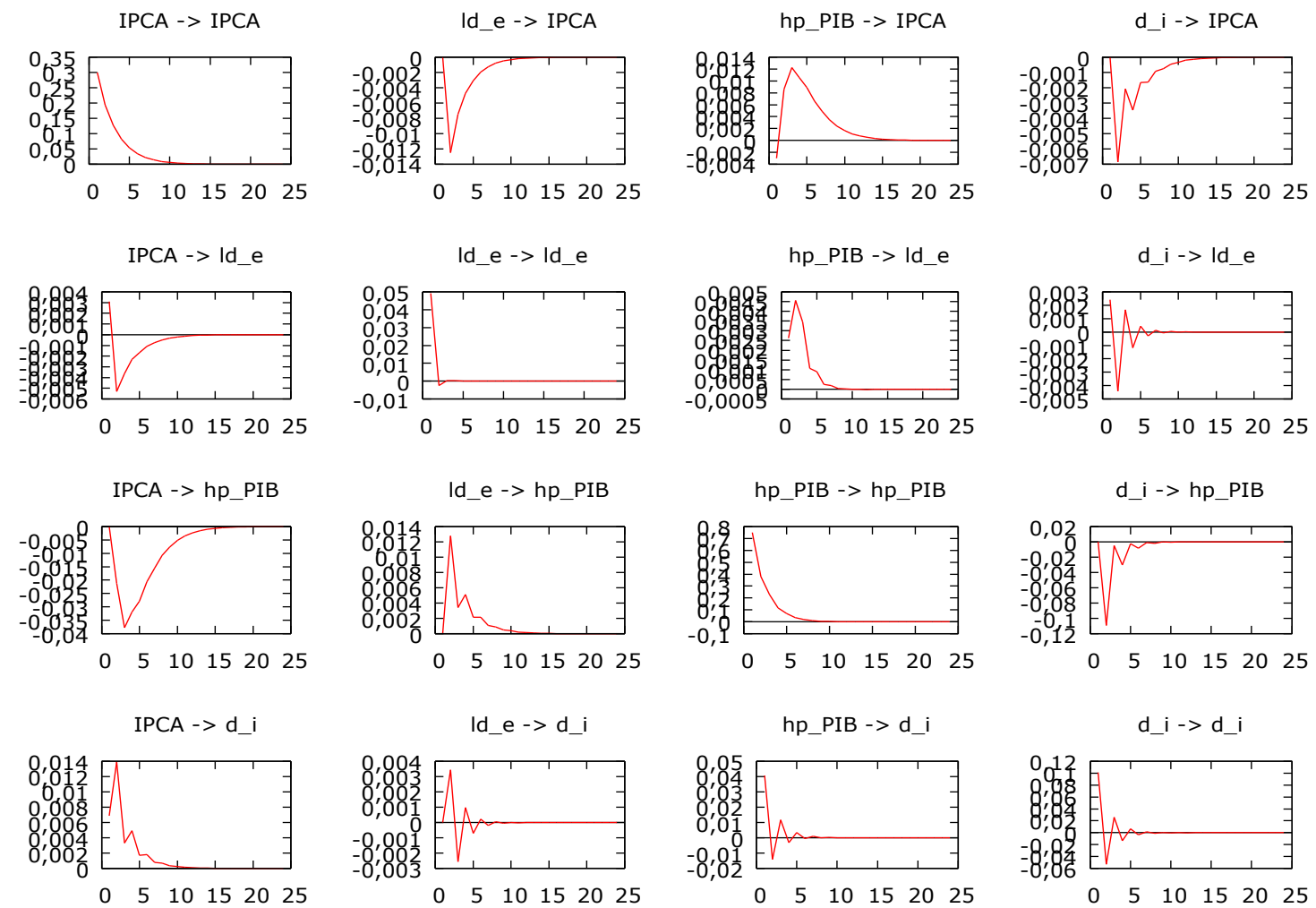

A análise dos gráficos da função impulso-resposta permite observar que uma elevação no diferencial de juros $(i)$, correspondente, por exemplo, a um aumento da SELIC em um contexto de taxas de juros internacionais constantes tem como efeito imediato uma redução na inflação medida pelo IPCA $(\pi)$. A redução na taxa de inflação abaixo de sua tendência é observada após 1 período e persiste até aproximadamente 10 períodos. Em relação ao câmbio $(e)$, a trajetória após o choque em $i$ apresenta oscilação, e o retorno ocorre após cerca de 5 períodos. Em relação ao hiato do produto $(y)$, os resultados apontam para valores negativos após o choque, persistindo o efeito até aproximadamente 7 períodos. Estes resultados podem ser considerados esperados no contexto do regime de metas de inflação.

Em relação à taxa de câmbio, observa-se uma imediata redução em IPCA. Neste caso, esperarse-ia uma elevação de IPCA, uma vez que a taxa de câmbio em elevação (desvalorização) torna os produtos importados relativamente mais caros, sejam eles insumos (neste caso pode-se argumentar que o efeito sobre a inflação seria após algum período de tempo) ou bens de consumo finais (neste caso diminui-se a competição do produto doméstico com o estrangeiro). No que diz respeito ao diferencial de juros, o efeito inicial é positivo, seguindo após 3 períodos uma trajetória oscilatória. Em relação ao hiato do produto, uma desvalorização cambial tem efeito positivo, que se estende por cerca de 10 períodos. 
Um choque em IPCA tem como efeito imediato elevação do diferencial dos juros, uma sensível elevação da inflação e uma tendência à redução no câmbio e no hiato do produto. No caso do hiato do produto, o efeito sobre a inflação é positivo e persiste por um longo período (superior a 10). O choque sobre o diferencial de juros apresenta comportamento oscilatório e os demais efeitos são positivos, com duração não superior a 10.

\section{Conclusões}

Neste trabalho realizamos um estudo empírico sobre o regime de metas de inflação, introduzindo a taxa de câmbio e considerando o diferencial de juros como forma de captar o efeito de variáveis ligadas ao setor externo da economia sobre o IPCA. Os resultados são coerentes com a teoria - o diferencial de juros positivo tende a reduzir o IPCA e o hiato do produto. No caso da taxa de câmbio, esta tem um efeito diferente do esperado em relação ao IPCA. A inclusão de novas variáveis ligadas ao setor externo da economia deve ser considerada em pesquisas futuras.

\section{Referências}

[1] Elaydi, S. "An introduction to difference equations". 3 rd ed. Springer, 2005.

[2] Enders, W. “Applied econometric time series”. New York: John Wiley \& Sons, 2004.

[3] Fraga et al.. Inflation targeting in emerging market economies. Working Paper - Banco Central do Brasil, $\mathrm{n}^{\circ}$ 76, 2003.

[4] Franco, G. H. B. "O Plano Real e outros ensaios". Rio de Janeiro: Francisco Alves, 1995.

[5] Gandolfo, G. "Economic Dynamics". Study Edition. Springer, 1997.

[6] Hamilton, J. T. “Time series analysis”. Princeton: Princeton University Press, 1994.

[7] Lima, G. T., Setterfield, M. Inflation Targeting and Macroeconomic Stability in a Post Keynesian Economy. Journal of a Post Keynesian Economics, Vol. 30 No. 3, pp. 435-461, Springer, (2008).

[8] Lobo, B. S., Oreiro, J. L. Metas de inflação, Crescimento e Estabilidade Macroeconômica: Uma análise a partir de um modelo Pós-keynesiano macrodinâmico não-linear. Anais do XXXIX Encontro Nacional de Economia (ANPEC), 2011.

[9] Minella, A. et al.. Inflation targeting in Brazil: Constructing credibility under exchange rate volatility. Working Paper - Banco Central do Brasil, n 77, (2003).

[10] Modenesi, A. M. "Regimes monetários: teoria e experiência do Real". Barueri: Manole, 2005.

[11] Modenesi, A. M., Araújo, E. C. Custos e benefícios do controle inflacionário no Brasil (20002008): uma análise empírica do mecanismo de transmissão da política monetária com base em modelo VAR. Anais do XXXVIII Encontro Nacional de Economia (ANPEC), 2010.

[12] Santos, A. L. M. Inflation targeting in a Post Keynesian economy. Journal of Post Keynesian Economics, Vol.4, No. 2 pp. 295-318, Winter, 2011-12.

[13] Sims, C. Macroeconomics and Reality. Econometrica, v. 58, p. 113-44, 1980. 